\title{
Electromagnetic pulse from final gravitational stellar collapse
}

\author{
P. D. Morley ${ }^{1}$ and I. Schmidt ${ }^{2}$ \\ 1 Development Systems Group B301, Veridian Systems Division, 14700 Lee Road, Chantilly, VA 20151, USA \\ e-mail: Peter.Morley@Veridian.com \\ 2 Departamento de Física, Universidad Técnica Federico Santa María, Casilla 110-V, Valparaíso, Chile \\ e-mail: ischmidt@fis.utfsm.cl
}

Received 14 May 2001 / Accepted 16 November 2001

\begin{abstract}
We employ an effective gravitational stellar final collapse model which contains the relevant physics involved in this complex phenomena: spherical radical infall in the Schwarzschild metric of the homogeneous core of an advanced star, giant magnetic dipole moment, magnetohydrodynamic material response and realistic equations of state (EOS). The electromagnetic pulse is computed both for medium size cores undergoing hydrodynamic bounce and large size cores undergoing black hole formation. We clearly show that there must exist two classes of neutron stars, separated by maximum allowable masses: those that collapsed as solitary stars (dynamical mass limit) and those that collapsed in binary systems allowing mass accretion (static neutron star mass). Our results show that the electromagnetic pulse spectrum associated with black hole formation is a universal signature, independent of the nuclear EOS. Our results also predict that there must exist black holes whose masses are less than the static neutron star stability limit.
\end{abstract}

Key words. pulsars: general - radiation mechanisms: general

\section{Introduction}

What is the measurable signal for gravitational collapse? It certainly produces gravitational waves, but unfortunately these have shown to be undetectable so far. However, there is another signal. Since the star has a sizable magnetic field, and since we expect this field to get quenched very rapidly during gravitational collapse, a massive electromagnetic pulse (MEMP) is created. We further show that the power spectrum is unlike any other naturally occurring phenomena: a wave packet whose wavetrain lasts only 2 milliseconds and whose characteristic power spectrum is a square block.

We present a simulation, within an effective model, of the iron-core collapse of massive stars which produce type II supernova. Depending on the core's mass, either a hydrodynamic bounce occurs above nuclear densities or the collapse races unhindered into the formulation of a stellar size black hole. Either case produces a MEMP. Our main contribution in this paper is the calculation of the energy spectrum of the MEMP. In cases where the collapsing core has mass greater than the dynamical neutron star mass limit (discussed herein), a hydrodynamic bounce cannot occur and no visible supernova results.

Send offprint requests to: P. D. Morley,

e-mail: pychen@rma.edu
In such cases, the MEMP is the only electromagnetic signal associated with the gravitational collapse.

One of the unexpected secondary results is the finding that, in general, there exist two classes of neutron stars: the first class is made up of solitary neutron stars which do not have the opportunity to accrete matter from a binary companion, and therefore have original masses coming from the collapse process. This mass is bounded by the ability of the nuclear equation of state to produce a hydrodynamic bounce. An unexpected secondary finding is that this imposes an heretofore unappreciated constraint on candidate nuclear equations of state. The maximum mass of the neutron star in this class is the dynamical mass, being the largest core mass that can undergo a hydrodynamic bounce. We will see that this dynamical mass limit is considerably less than the static mass limit, so the bounce does indeed save the core from further collapse due to its own self-gravity. The second class of neutron stars are those that can accrete matter from a binary companion and so their maximum mass is constrained by the requirement of static stability: the maximum static neutron star mass. In general, we find that the maximum static mass can exceed the dynamic mass by as large as $100 \%$.

A current review of the status of gravitational collapse is given in Joshi (2000), who discusses spherically symmetric collapse. Fryer \& Heger (2000) discuss core collapse from the viewpoint of creating type II supernova 
from the hydrodynamic bounce. The remnant size cores found by Fryer and Heger are close to the sizes in this paper, though they do not investigate the remnant size versus the nuclear equation of state. In the present paper, we consider spherical radial infall and stop the simulation if bounce occurs; continuing on would require incorporating shock physics. As shown in the work of Bocquet et al. (1995), only gigantic magnetic fields $B>10^{10} \mathrm{~T}=10 \mathrm{GT}$ $\left(\right.$ Testla $=10^{4}$ Gauss, GT $\left.=10^{9} \mathrm{~T}\right)$ cause stellar deformation. Since the magnetic fields considered in this paper fall far below this large critical field, the star is not deformed by its magnetic field. An interesting followup to the research presented here, is to apply the effective gravitational collapse model to stars having non-negligible rotation.

In general, there are three sources for electromagnetic and gravitational radiation associated with stellar collapse: the direct radiation phase emitted by the stellar object before the formation of a black hole, the socalled damped oscillations known as quasi-normal ringing (Iyer 1987) that are the vibrations of a black hole shaking off its non-zero multipole charge moments, and the late-time power-law tail (Price 1972; Cunningham 1978; Leaver 1986; Hod 1999, 2000a,b) underneath the damped oscillations. Because of the gravitational redshift, essentially the only energy that survives to infinity comes from the direct radiation phase, before the black hole forms. In this paper, we calculate the direct phase electromagnetic energy radiated by stellar objects that bounce and become stable neutron stars, and stellar objects so massive that they become black holes.

In order to calculate the electromagnetic radiation produced by the gravitational collapse, our strategy is the following. During the collapse the infall kinetic energy decreases while the system does mechanical work against the internal star pressure. This pressure has, in general, two components: material (nuclear) pressure and electromagnetic pressure. However, all published nuclear equations of state have the property that the nuclear pressure is orders of magnitude larger than the electromagnetic pressure; indeed, only for magnetic fields greater than $10^{18}$ Gauss does the electromagnetic pressure approach parity with the nuclear pressure. Using conservation of energy, the continuity equation and the material equation of state, we can calculate how the star radius changes with time. If we further assume that the stellar magnetic field can be approximated by a dipole field, and that the lines of magnetic field are frozen in the material and carried along with it, we can relate the change in the star's radius with time to the change in its effective magnetic moment. The usual dipole radiation formula then gives the energy spectrum of the radiation.

\section{The effective gravitational collapse model}

We simulate the collapse to the hydrodynamic bounce, or to twice the gravitational radius, $a_{\mathrm{g}}=2 G M / c^{2}$, if no bounce occurs. In the former case, the bounce cuts

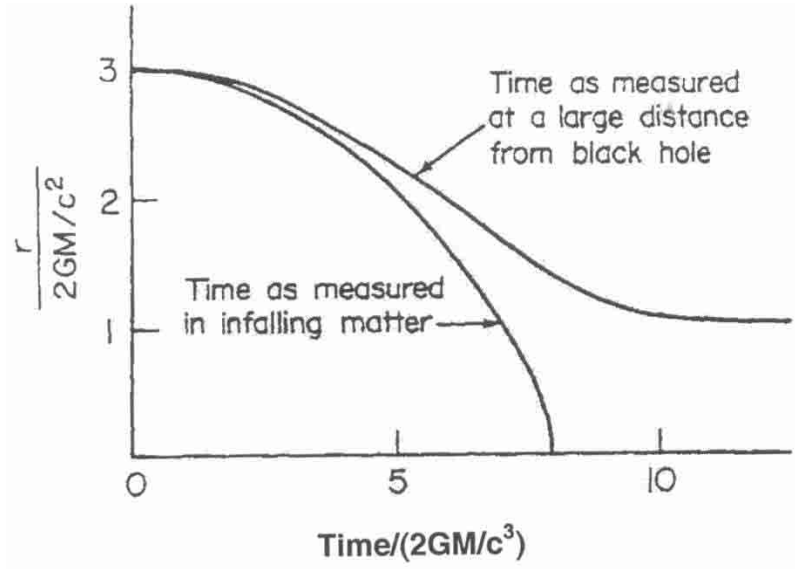

Fig. 1. Difference between proper and Schwarzschild times.

off the MEMP, while in the latter case, final black hole formation at radius $=a_{\mathrm{g}}$ occurs almost instantaneously later after the simulation ends. Because of the gravitational redshift, the final stellar trajectory from $2 a_{\mathrm{g}}$ to $a_{\mathrm{g}}$ adds little to the observable energy at infinity. Thus the MEMP calculated is expected to be an accurate profile. The reason why we stop at $2 a_{\mathrm{g}}$ is because, while the Schwarzschild metric is the exterior metric during gravitational collapse and as such, is the clock time in the effective collapse model, the radiation process must be calculated in proper time. In Fig. 1, we plot the difference between proper time and Schwarzschild time. Only when the core radius becomes less than $3 a_{\mathrm{g}}$ is there a difference in the two clocks. By stopping the simulation at $2 a_{\mathrm{g}}$, we can still reliably calculate the core surface as a function of proper time, by using the Taylor expansion employing the Schwarzschild time solution of the collapse model. Indeed, one can calculate from this figure that the time error is 8.364 microseconds $\times X$, where $\mathcal{M} / \mathcal{M}_{\odot}=X$, with $\mathcal{M}$ being the core mass. At the same time, we fold in the gravitational and Doppler redshift, since the difference between the Schwarzschild time and proper time leads to a gravitational redshift of the produced radiation.

The collapse process from the initial starting configuration of the iron-core mass density $(\rho)$ of $\rho=1 \times$ $10^{12} \mathrm{gm} / \mathrm{cm}^{3}$ and inward speed $v / c \sim 2 / 3 \times 10^{-2}$ to either hydrodynamic bounce or twice $a_{\mathrm{g}}$ takes less than 3 milliseconds. In contrast, the characteristic time scale for neutrino diffusion out of the core is $\sim 2 \rightarrow 10 \mathrm{~s}$. This $10^{3}$ times larger neutrino diffusion scale means that these particles cannot influence the bounce or the MEMP. The role of the neutrinos is not to carry away the binding energy of the collapsing matter, as it sometimes is erroneously stated. Rather, the collapsing matter must do work against the equation of state and this energy is stored as internal energy, entirely analogous to a weight compressing a spring. This stored energy is not lost, but remains primarily as elevated Fermi levels of electrons and nucleons. Thus the energy the neutrinos actually carry away is the energy from nuclear beta decay, relaxed elastic energy and thermal heat energy. The difference in the time scales for 


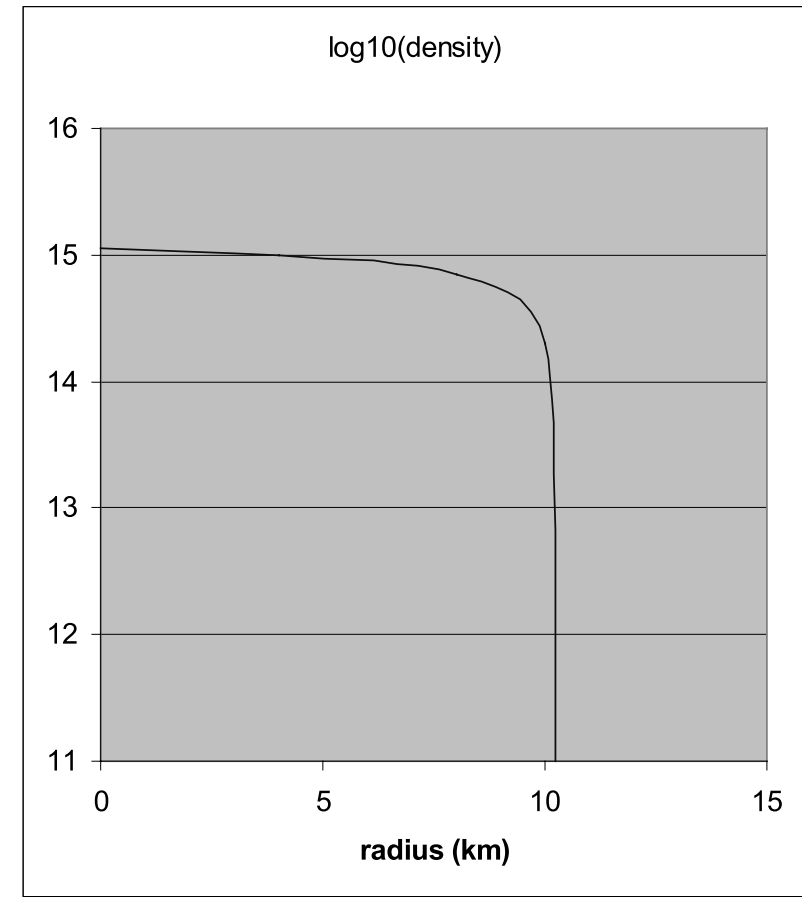

Fig. 2. Neutron star density profile using the FriedmanPandharipande equation of state, Straumann (1992).

neutrino diffusion and hydrodynamic infall means that the iron-core collapse is adiabatic, allowing a concise formulation for the conservation of energy. Previous work, Morley (2000), showed that neutrino transport in the iron-core was due to elastic scattering and typically $\sim 1000$ collisions occurred before escape. Our collapse code in the present paper shows beyond a doubt, that if no hydrodynamic bounce occurs, these diffusing neutrinos are trapped in the infalling nuclear material and are carried into the resulting black hole. Thus observation of neutrinos associated with particular supernova proves that the initial infall did not form a black hole.

Another physical insight into the problem is the recognition that the iron-cores have flat density profiles. In Fig. 2, we show the neutron star density profile using the Friedman-Pandharipande equation of state, Straumann (1992). Thus the approximation that the star collapses homogeneously is excellent ( $t=$ Schwarzschild time): $\rho(\boldsymbol{r}, t)=\rho(t)$. In this paper, we treat spherical radial infall. As mentioned earlier, a very interesting follow-on to this research is to remove this constraint and allow core rotation in the effective collapse model.

Further simplifications can be achieved by using the fact that the electrical conductivity of the iron-core is very high, nearly infinite. This allows the magnetohydrodynamic approximation: the lines of magnetic force are frozen into the material and are carried along with it. In this paper, we approximate the iron-core stellar magnetic field by a magnetic dipole of moment $\boldsymbol{m}$. We use the model

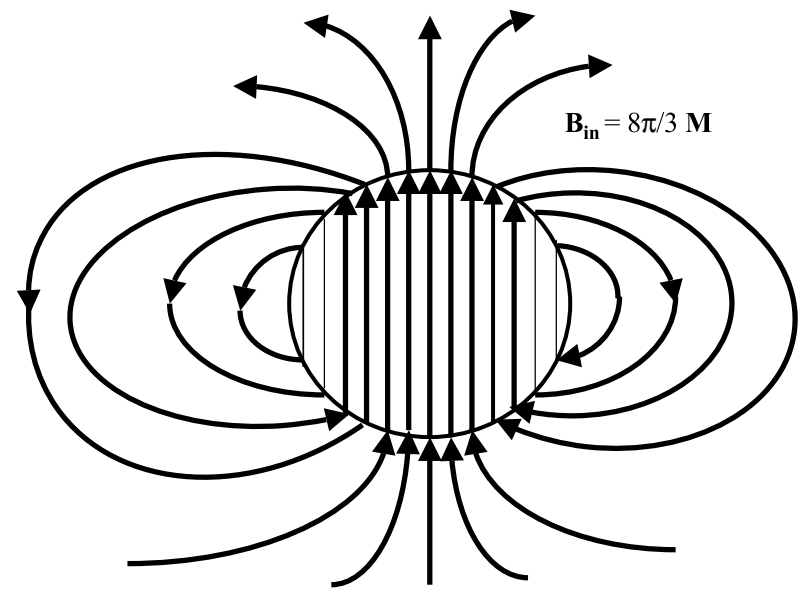

Fig. 3. Stellar magnetic dipole field.

of a uniformly magnetized sphere of magnetization per unit volume $\boldsymbol{M}$. The relation of $\boldsymbol{m}$ to $\boldsymbol{M}$ is

$\boldsymbol{m}=\frac{4 \pi}{3} a^{3} \boldsymbol{M}$

where $a$ is the stellar radius. $\boldsymbol{B}_{\text {out }}=\boldsymbol{H}$ outside the sphere and is a magnetic dipole field:

$\boldsymbol{B}_{\text {out }}=\frac{4 \pi}{3} a^{3}|\boldsymbol{M}|\left\{\frac{2 \cos \theta}{r^{3}} \hat{r}+\frac{\sin \theta}{r^{3}} \hat{\theta}\right\}$

while inside the core

$\boldsymbol{B}_{\text {in }}=\frac{8 \pi}{3} \boldsymbol{M}$.

This is shown in Fig. 3. In general, $a=a(t)$ and $\boldsymbol{M}=$ $\boldsymbol{M}(t)$. The magnetohydrodynamic material response is the constancy of the magnetic flux through the polar region, Fig. 4

$\pi a^{2}\left(t_{1}\right) \sin ^{2} \theta B_{\text {in }}\left(t_{1}\right)=\pi a^{2}\left(t_{2}\right) \sin ^{2} \theta B_{\text {in }}\left(t_{2}\right)$,

(where $\left|\boldsymbol{B}_{\text {in }}\right|=B_{\text {in }}$ ), giving

$a^{2}\left(t_{1}\right)\left|\boldsymbol{M}\left(t_{1}\right)\right|=a^{2}\left(t_{2}\right)\left|\boldsymbol{M}\left(t_{2}\right)\right|=$ constant $=C_{m}$.

Therefore

$\boldsymbol{m}=\frac{4 \pi}{3} a C_{m} \hat{z}$

and

$\ddot{m}(t)=\frac{4 \pi}{3} C_{m} \ddot{a}(t), m=|\boldsymbol{m}|$.

$C_{m}$ is fixed by astrophysical data. Young pulsars (e.g. PSR B0154+61) have the polar field strength $B \cong 2 \mathrm{GT}$. Since this is over a flux area of $10 \times 10 \mathrm{~km}^{2}$, the constant is $C_{m} \simeq 8 \times 10^{25} \mathrm{~cm}^{2}$. Gauss.

Thus we have obtained a relation between the second order time derivative of the stellar effective magnetic moment and the star's surface acceleration, which will allow us to obtain the power radiated once we know the acceleration. This will be obtained by a numerical simulation, whose ingredients we explain in the next sections. 


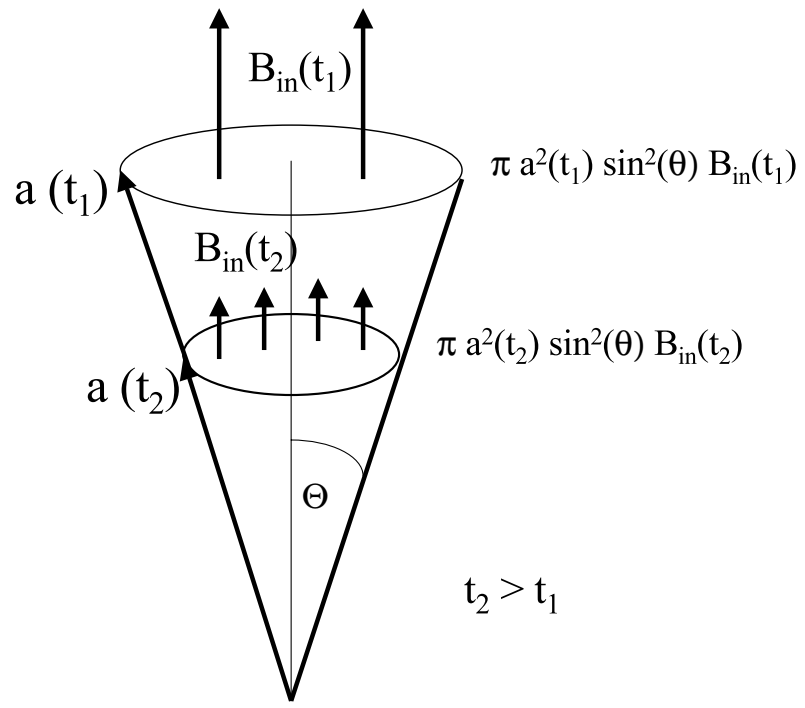

Fig. 4. Constancy of the magnetic flux through the polar region.

\subsection{Conservation of matter}

The conservation of baryon number is given by the equation

$\frac{\mathrm{d} n_{\mathrm{bar}}}{\mathrm{d} \tau}=-n \nabla \cdot u$

where $n_{\text {bar }}$ is the baryon density, $\tau$ is proper time, $\nabla$ is the 4-derivative and $u$ is the 4 -velocity. As long as $\tau \simeq t$ and extreme relativistic speeds are not encountered, then the conservation of baryon number reduces to the conservation of matter:

$\boldsymbol{\nabla} \cdot(\rho \boldsymbol{v})+\frac{\partial \rho}{\partial t}=0$

The only region in the collapse phenomena where the two conservation laws begin to differ is near twice $a_{\mathrm{g}}$. Though we treat $\rho$ as uniform throughout the spherical volume, it is changing in time $t$ so $\frac{\partial \rho}{\partial t}=\frac{\mathrm{d} \rho}{\mathrm{d} t} \neq 0$. Thus $\boldsymbol{v}$ cannot be a constant. With $\boldsymbol{\nabla} \cdot(\rho \boldsymbol{v})=\rho \boldsymbol{\nabla} \cdot \boldsymbol{v}=\frac{\rho}{r^{2}} \frac{\mathrm{d}}{\mathrm{d} r}\left(r^{2} v_{r}\right)$ where $\boldsymbol{v}=v_{r} \hat{r}$, the conservation of matter equation becomes

$\frac{\mathrm{d} \rho}{\mathrm{d} t}+\frac{\rho}{r^{2}} \frac{\mathrm{d}}{\mathrm{d} r}\left(r^{2} v_{r}\right)=0$.

This has the physical solution

$v_{r}=-\frac{r}{3 \rho} \frac{\mathrm{d} \rho}{\mathrm{d} t}$.

\subsection{Conservation of energy}

The energy $E_{\text {if }}$ available to do work if the matter collapses from mass density $\rho_{\mathrm{i}}$ to $\rho_{\mathrm{f}}$ is

$E_{\text {if }}=P E_{\mathrm{i}}+K E_{\mathrm{i}}-P E_{\mathrm{f}}$

where $K E_{\mathrm{i}}, K E_{\mathrm{f}}$ is the kinetic energy at the initial, final density respectively, and $P E_{\mathrm{i}}, P E_{\mathrm{f}}$ is the gravitational self-energy of the core with initial, final mass density respectively. The work $\mathcal{W}>0$ done against the equation of state in compressing the matter is

$\mathcal{W}=-\int_{V_{\mathrm{i}}}^{V_{\mathrm{f}}} P \mathrm{~d} V, P=$ pressure.

Here $V_{\mathrm{i}}, V_{\mathrm{f}}$ are the initial and final core volumes respectively. The core will bounce when or if it reaches a density such that

$E_{\text {if }}=\mathcal{W}$

For realistic equations of state, we expect a bounce for core masses of the order of a solar mass.

\subsection{Gravitational self-energy}

A core of radius $a$ in the Schwarzschild metric has gravitational potential energy, Weinberg (1972)

$P E=\int_{0}^{a} 4 \pi r^{2}\left\{1-\frac{1}{\sqrt{1-\frac{2 G \mathcal{M}(r)}{c^{2} r}}}\right\} \rho_{\mathrm{e}} \mathrm{d} r$

where $c$ is speed of light, $\rho_{\mathrm{e}}$ is the energy density of matter and $\mathcal{M}(r)$ is the amount of mass within radius $r$. Since $\mathcal{M}(r)=\frac{r^{3}}{a^{3}} \mathcal{M}$ and $\rho_{\mathrm{e}}=\frac{3 \mathcal{M} c^{2}}{4 \pi a^{3}}$, this reduces to

$P E=\rho_{\mathrm{e}} \int_{0}^{a} 4 \pi r^{2}\left\{1-\frac{1}{\sqrt{1-\frac{2 G \mathcal{M} r^{2}}{c^{2} a^{3}}}}\right\} \mathrm{d} r$

which can be integrated to give

$P E=\mathcal{M} c^{2}+\frac{2 \pi \rho_{\mathrm{e}} a}{\kappa}\left\{\sqrt{1-\kappa a^{2}}-\frac{1}{a \sqrt{\kappa}} \arcsin a \sqrt{\kappa}\right\}$

where $\kappa=\frac{2 G \mathcal{M}}{c^{2} a^{3}}, G=$ gravitational constant.

\subsection{Kinetic energy of infall}

The equation of state (EOS) of matter (to be discussed in more detail later) can describe "hot" or "cold" nuclear matter. In general, the thermal energies that are reached in the collapse phenomena are a small fraction of the gravitational binding energy experienced by the particles. If the pressure $P$ in Eq. (13) describes a "hot" EOS, then some of the kinetic energy + potential energy of infall is turned into heat. The numerical procedure (to be discussed in detail in a later section) takes small time slices $(\Delta t)$ and computes the new kinetic energy after work against the EOS is determined. The equation is

$K E(t+\Delta t)=$

$K E(t)+P E(t)-P E(t+\Delta t)+\int_{V(t)}^{V(t+\Delta t)} P \mathrm{~d} V$.

Thus the effective gravitational model takes into account any desired heat generation through the inputed EOS. 
We need the instantaneous kinetic energy of a collapsing iron-core in the Schwarzschild metric. We want the expression to be accurate to $\mathrm{O}\left(v_{r}^{4} / c^{4}\right)$.

To order $\mathrm{O}\left(v_{r}^{2} / c^{2}\right)$, the instantaneous kinetic energy is

$K E=\int_{0}^{a} 4 \pi r^{2}\left(\frac{\rho_{\mathrm{e}}(r)}{2}\right) \frac{v_{r}^{2}(r)}{c^{2}} \frac{1}{\sqrt{1-\frac{2 G \mathcal{M}(r)}{c^{2} r}}} \mathrm{~d} r$

where

$v_{r}^{2}(r)=r^{2}\left(\frac{1}{3 \rho} \frac{\mathrm{d} \rho}{\mathrm{d} t}\right)^{2}=r^{2} \mathcal{B}, \mathcal{B}=\left(\frac{1}{3 \rho} \frac{\mathrm{d} \rho}{\mathrm{d} t}\right)^{2}$.

This becomes

$K E=2 \pi \rho_{\mathrm{e}} \frac{\mathcal{B}}{c^{2}} \int_{0}^{a} \frac{r^{4} \mathrm{~d} r}{\sqrt{1-\kappa r^{2}}}$.

Computing this integral:

$$
\begin{aligned}
K E= & 2 \pi \rho_{\mathrm{e}} \frac{\mathcal{B}}{c^{2}}\left\{-\frac{a^{3}}{4 \kappa} \sqrt{1-\kappa a^{2}}-\frac{3}{8 \kappa^{2}} a \sqrt{1-\kappa a^{2}}\right. \\
& \left.+\frac{3}{8 \kappa^{5 / 2}} \arcsin a \sqrt{\kappa}\right\} .
\end{aligned}
$$

The kinetic energy to $\mathrm{O}\left(v_{r}^{4} / c^{4}\right)$ is

$K E=$

$\int_{0}^{a} 4 \pi r^{2}\left(\frac{\rho_{\mathrm{e}}(r)}{2}\right)\left[\frac{v_{r}^{2}(r)}{c^{2}}-\frac{1}{8} \frac{v_{r}^{4}(r)}{c^{4}}\right] \frac{1}{\sqrt{1-\frac{2 G \mathcal{M}(r)}{c^{2} r}}} \mathrm{~d} r$

This extra piece can be integrated in the same manner as the first piece, and using the parameters $\dot{a}$ (speed of surface) and $\lambda=\kappa a^{2}$ we find that the kinetic energy to $\mathrm{O}\left(v_{r}^{4} / c^{4}\right)$ is

$$
\begin{aligned}
K E= & \mathcal{M} c^{2}\left\{\frac { 3 } { 2 } ( \frac { \dot { a } } { c } ) ^ { 2 } \frac { 1 } { \lambda ^ { 5 / 2 } } \left[-\frac{1}{4} \lambda^{3 / 2} \sqrt{1-\lambda}\right.\right. \\
& \left.-\frac{3}{8} \sqrt{\lambda} \sqrt{1-\lambda}+\frac{3}{8} \arcsin \sqrt{\lambda}\right] \\
& +\frac{15}{2}\left(\frac{\dot{a}}{c}\right)^{4} \frac{1}{\lambda^{7 / 2}}\left[\sqrt { 1 - \lambda } \left(\frac{\sqrt{\lambda}}{64}+\frac{\lambda^{3 / 2}}{96}\right.\right. \\
& \left.\left.+\frac{\lambda^{5 / 2}}{120}\right)-\frac{1}{64} \arcsin \sqrt{\lambda}\right] .
\end{aligned}
$$

\subsection{Nuclear Equation of State (EOS)}

The hydrodynamic bounce is independent of the equation of state below nuclear density (nuclear density $\simeq 2 \times$ $10^{14} \mathrm{gm} / \mathrm{cm}^{3}$ ). We know this because if we put in zero pressure in Eq. (13) below nuclear density, the bounce properties hardly change at all. The hot thermal gas kinetic EOS below nuclear density is not responsible for the hydrodynamic bounce, and this can be shown by calculating the work $\mathcal{W}$. In this paper, we use two completely different nuclear EOS, and while the contribution of the pressure below nuclear densities is negligible, we still hook them to EOS of degenerate electronic matter used frequently in collapse calculations (Baym 1971).
Table 1. The three regimes for the nuclear EOS. The first and third are in beta decay equilibrium, but the middle is out of equilibrium. Here $N=$ the number of neutrons and $Z=$ the number of protons.

\begin{tabular}{||c|c|c||}
\hline Class & Property & Applicability \\
\hline normal matter & $N \simeq Z$ & atomic nuclei \\
iron-core & $N \simeq 2 Z$ & proto-neutron star \\
neutronic & $N \gg>Z$ & cold neutron star \\
\hline
\end{tabular}

The different regimes for the nuclear EOS are listed in Table 1.

In this paper, two different nuclear EOS were used in the collapse calculations: the "Argonne AV14 + UVII" (Wiringa 1988) and the QCD (Kislinger 1978).

Perturbation theory in quantum chromodynamics (QCD) breaks down at twice nuclear density, where it must be joined to phenomenological EOS. Both EOS used here are joined to the Baym EOS, as mentioned earlier. At some time in the future, the nuclear EOS will be known accurately, and then collapse calculations will lead to predictions with less error.

Now we have all the ingredients for the numerical simulation. Once the initial stellar radius, surface velocity, mass and density are given, we can compute $\mathrm{d} \rho / \mathrm{d} t$ using the continuity Eq. (11), and therefore the new density and potential energy (Eq. (17)) in the next $\Delta t$ step. Then we calculate the work necessary to reach this new density using the corresponding EOS, and check whether the bounce condition given in Eq. (14) is met. If it is, which means that the new kinetic energy vanishes, there is a bounce and we stop the simulation. If it is not, we continue by checking also whether we have reached twice $a_{\mathrm{g}}$, in which case we also stop. If we have not reached that radius we compute the new kinetic energy (18), which allows us in turn to get the new surface velocity solving Eq. (24) and then use the continuity equation again, and so on. This logic is described in Fig. 5. Notice that the desired quantity, the surface acceleration, is obtained directly from the continuity Eq. (11), as

$\ddot{a}=\frac{4}{9} \frac{a}{\rho^{2}}\left(\frac{\mathrm{d} \rho}{\mathrm{d} t}\right)^{2}-\frac{a}{3 \rho} \frac{\mathrm{d}^{2} \rho}{\mathrm{d} t^{2}}$.

\section{Radiation}

The MEMP calculated here is magnetic dipole radiation; it is not the emission of a black-body at some temperature and radius. The MEMP, while it exists, completely swamps thermal emission. Our goal is to calculate the MEMP energy spectrum. The energy radiated in all directions per unit time, in Gaussian units, is (Landau 1975):

$\frac{\mathrm{d} E}{\mathrm{~d} \tau}=\frac{2}{3 c^{3}}|\ddot{m}(\tau)|^{2}$.

We introduce the Fourier transforms $m(\omega)$

$m(\tau)=\frac{1}{\sqrt{2 \pi}} \int m(\omega) \mathrm{e}^{i \omega \tau} \mathrm{d} \omega$ 
1. Set initial a, da/dt, $\mathbf{M}_{\text {core }}, \rho$

2. Compute $\mathrm{KE}$

3. Compute $\mathbf{d} \rho / \mathbf{d t}$ and $\rho_{\text {new }}$ in next $\Delta \mathrm{t}$ time step

4. Compute potential energy change

5. Compute work necessary to reach $\rho_{\text {new }}$

6. Check if step $5>\operatorname{step} 4+\operatorname{step} 2$

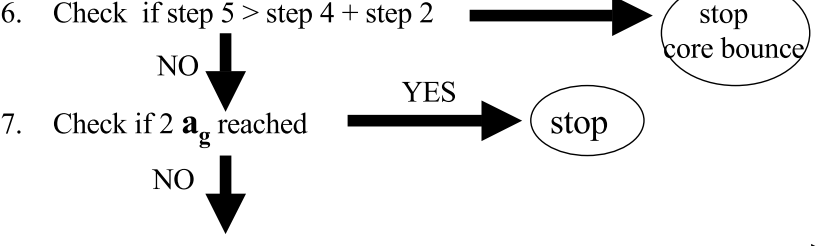

7.

8. Compute $\mathrm{KE}$ of infall

Fig. 5. Code logic.

$m(\omega)=\frac{1}{\sqrt{2 \pi}} \int m(\tau) \mathrm{e}^{-i \omega \tau} \mathrm{d} \tau$

Then

$\ddot{m}(\tau)=-\frac{1}{\sqrt{2 \pi}} \int \omega^{2} m(\omega) \mathrm{e}^{i \omega \tau} \mathrm{d} \omega$.

Taking the integration over time from $-\infty$ to $+\infty$ gives the total energy radiated

$E=\frac{2}{3 c^{3}} \int \omega^{4}|m(\omega)|^{2} \mathrm{~d} \omega$

and the energy spectrum

$\frac{\mathrm{d} E}{\mathrm{~d} \omega}=\frac{2}{3 c^{3}} \omega^{4}|m(\omega)|^{2}$.

\subsection{Roll-on-roll-off (RORO) function}

The core's dipole moment is

$m(\tau)=\frac{4 \pi}{3} C_{m} a(\tau)$

where $a(\tau)$ is the core's radius in $\mathrm{km}$ at proper time $\tau$. As discussed in the later sections, we fit $a(\tau)$ to a polynomial curve and compute $\ddot{m}(\tau)$. Then we integrate analytically to obtain the total energy release. To know the energy spectrum, though, and to include the Doppler and gravitational redshifts, we must compute the Fourier transform. If we put in Heaviside functions in time for the beginning and ending of the infall, the Fourier transform will not give rise to a finite energy spectrum because the derivative of a step function is a delta function. Physically we must impose the following boundary conditions: from time $-\infty$ we had the initial radius and at time $+\infty$ we have the final radius, and smooth transition between them in the infall period. The analogous problem occurs in particle scattering. Thus we need a Roll-on-Roll-off (RORO) function to accomplish this mathematically. Any suitable RORO function will do and gives the same answer. Thus the time dependent $m(\tau)$ becomes (with $C_{0}=\frac{4 \pi}{3} C_{m}$ )

$$
\begin{aligned}
& m(\tau) \Rightarrow C_{0} \frac{1}{2} a_{\max }\left(1-\tanh \left(\frac{\tau}{\mu \mathrm{s}}\right)\right) \\
& +.25\left(1+\tanh \left(\frac{\tau}{\mu \mathrm{s}}\right)\right) \times\left(1-\tanh \left(\frac{\tau-T}{\mu \mathrm{s}}\right)\right) m(\tau) \\
& \quad+C_{0} \frac{1}{2} a_{\min }\left(1+\tanh \left(\frac{\tau-T}{\mu \mathrm{s}}\right)\right) .
\end{aligned}
$$

In the above equation, $m(\tau)$ on the right-hand-side is the fitted polynomial function in proper time $\tau$ (fifth order polynomial - fitted from the collapse code output of the acceleration), $\tau$ and $T=$ collapse time, are in microseconds, and $a_{\max }$ and $a_{\min }$ are respectively the starting and ending core radius in $\mathrm{km}$. Any derivation of the gravitational or electromagnetic power spectrum must use a RORO function. As we will see in the following sections, the RORO function is also critical to deriving the redshifts.

\subsection{Total energy}

We fit the acceleration collapse solution to a polynomial function

$a(\tau)=\mathrm{km} \sum_{i=0}^{5} b(i)(\tau / \mu \mathrm{s})^{i}$

where $\tau$ is in microseconds. Then

$m(\tau)=C_{0} \mathrm{~km} \sum_{i=0}^{5} b(i)(\tau / \mu \mathrm{s})^{i}$

and

$$
\begin{aligned}
|\ddot{m}(\tau)|^{2}= & C_{0}^{2} \mathrm{~km}^{2}\left(\frac{1}{\mu \mathrm{s}}\right)^{4} \sum_{i=2}^{5} b(i)\left(\frac{\tau}{\mu \mathrm{s}}\right)^{i-2} \\
& \times \sum_{j=2}^{5} b(j)\left(\frac{\tau}{\mu \mathrm{s}}\right)^{j-2} \cdot
\end{aligned}
$$

The total energy emitted in the direct phase of the collapse is then

$$
\begin{aligned}
E= & \frac{2 C_{0}^{2} \mathrm{~km}^{2}}{3 c^{3}}\left(\frac{1}{\mu \mathrm{s}}\right)^{3} \\
& \times \sum_{i=2}^{5} \sum_{j=2}^{5} b(i) b(j)\left(\frac{T}{\mu \mathrm{s}}\right)^{i+j-3} \frac{1}{i+j-3}
\end{aligned}
$$

The $b(i)$ are fitted by a least-squares program. $T$ is the collapse time in microseconds.

When the collapse extends below $3 a_{\mathrm{g}}$, we need to use multiple RORO functions to separate out the contribution of each surface to the radiation spectrum, since each surface has a different gravitational redshift and Doppler shift. Then the energy radiated from each surface radius can be corrected by the correct attenuation factor, as perceived from an observer far away. 


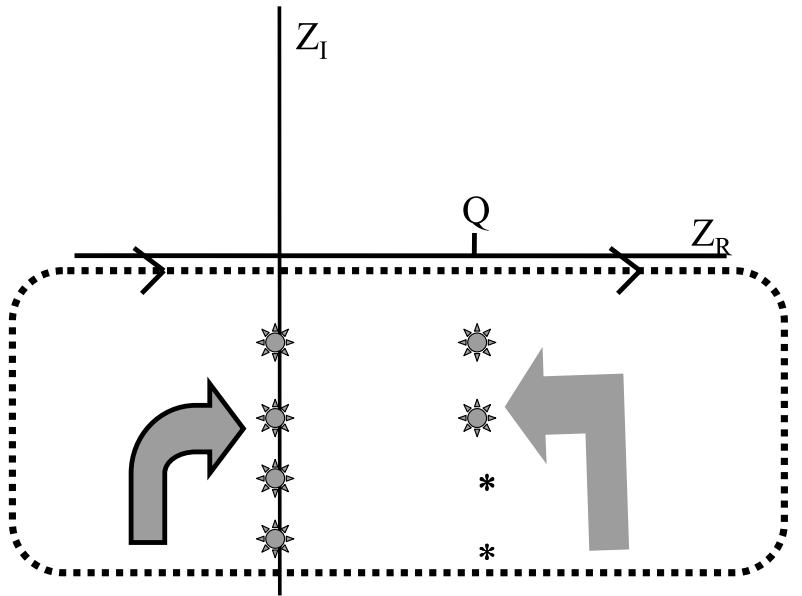

Poles@ $\mathrm{Z}=-\mathrm{i}(2 \mathrm{n}+1) / 2$

Poles @ $\mathrm{Z}=\mathrm{Q}-\mathrm{i}(2 \mathrm{n}+1) / 2$

Fig. 6. Integration contour.

\subsection{Fourier transform}

We must compute $m(\omega)$ where $m(\omega)$ is given by Eq. (28). Letting $s \equiv \omega \cdot \mu \mathrm{s}, x \equiv \tau / \mu \mathrm{s}$ and $Q \equiv T / \mu \mathrm{s}$, we have

$$
\begin{aligned}
m(\omega)= & \mu \mathrm{s} \operatorname{km} C_{0} \frac{1}{\sqrt{2 \pi}} \int_{-\infty}^{+\infty} \mathrm{d} x \mathrm{e}^{-i s x}\left\{.5 \frac{a_{\max }}{\mathrm{km}}(1-\tanh x)\right. \\
& +.25(1+\tanh x)(1-\tanh (x-Q)) \sum_{i=0}^{5} b(i) x^{i} \\
& \left.+.5 \frac{a_{\min }}{\mathrm{km}}(1+\tanh (x-Q))\right\} .
\end{aligned}
$$

This integral can be done using contour integration in the complex energy plane. Letting $x=Z_{r}+i Z_{i}$ (complex), we have damping for $Z_{i}<0$. Poles in the lower half of the complex plane occur at $Z_{r}=0, Z_{i}=-\frac{2 N+1}{2} \pi$ and at $Z_{r}=Q, Z_{i}=-\frac{2 N+1}{2} \pi$. We integrate clockwise along the contour of Fig. 6 to obtain the answer:

$$
\begin{aligned}
& m(\omega)=\frac{\mu \mathrm{s} \mathrm{km} C_{0}}{\sqrt{2 \pi}}(-2 \pi i)\left\{\sum _ { N = 0 } ^ { \infty } \mathrm { e } ^ { - s \pi \frac { 2 N + 1 } { 2 } } \left[-.5\left(a_{\max } / \mathrm{km}\right)\right.\right. \\
& \left.+.25 \frac{1+\tanh Q}{\tanh Q} \sum_{j=0}^{5} b(j)\left(-i \pi\left(\frac{2 N+1}{2}\right)\right)^{j}\right] \\
& +\sum_{N=0}^{\infty} \mathrm{e}^{-i s Q-s \pi\left(\frac{2 N+1}{2}\right)}\left[-.25 \frac{1+\tanh Q}{\tanh Q} \cdot \sum_{j=0}^{5} b(j) \cdot\right. \\
& \left.\left.\left(Q-i \pi\left(\frac{2 N+1}{2}\right)\right)^{j}+.5\left(a_{\min } / \mathrm{km}\right)\right]\right\}
\end{aligned}
$$

The presence of the exponentials signals that the integration over $\omega$ in Eq. (30) is indeed finite.

\subsection{Redshift}

The difference between the proper time and the Schwarzschild time only occurs when the radius is below three times $a_{\mathrm{g}}$. In order to compute the gravitational and

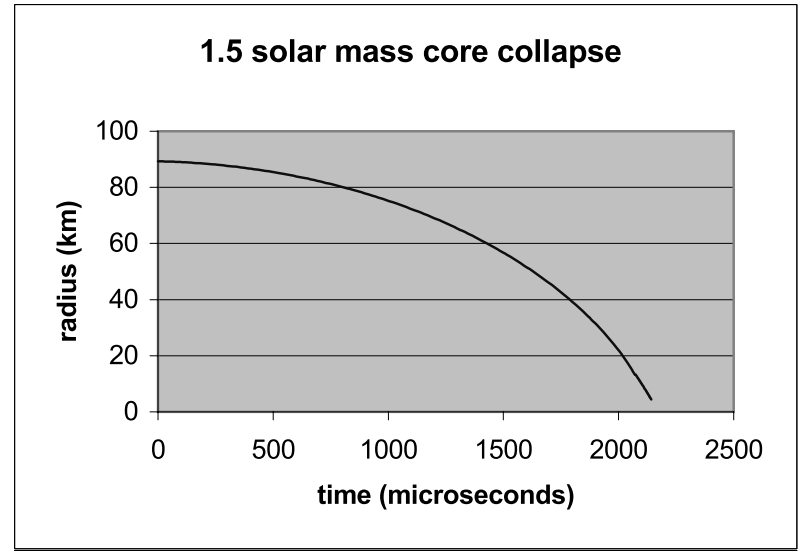

5 Solar Mass Core Collapse
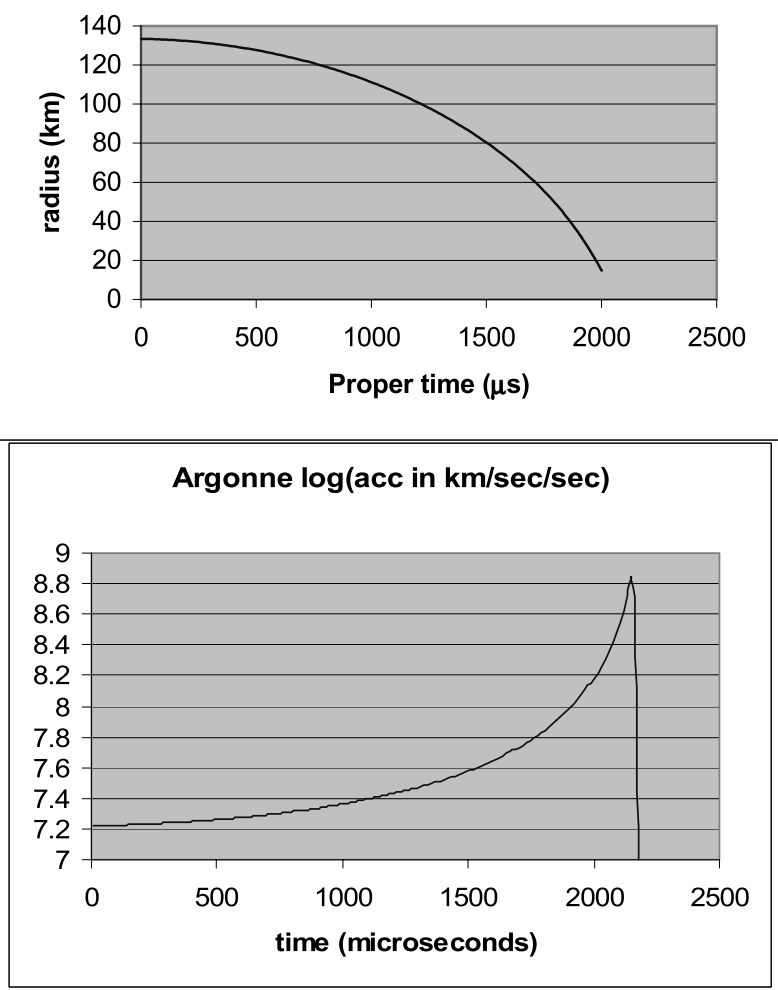

Fig. 7. Radii time trajectory, for core masses of 1.5 and 5 solar masses, for the initial conditions stated in the text. Also, an acceleration profile of a core bounce in the Argonne EOS, for a 0.75 solar mass core.

Doppler redshifts, we need to separate out the contribution of each accelerated surface to the radiation spectrum. This is done by the use of the RORO function. Consider the first RORO function from $\tau=0$ to $\tau=\tau_{1}$, and the second RORO function from $\tau=\tau_{1}$ to $\tau=\tau_{2}$. One can show that the complex $\tau_{1}$ poles of these two RORO functions mutually cancel in their sum, so the sum of two RORO reduces to one RORO from $\tau=0$ to $\tau=\tau_{2}$. By breaking the collapse into discreet radii using the RORO function, one can fold into the spectrum both the gravitational and Doppler redshift using the fact that each accelerated radius contributes incoherently to the power spectrum. 
Table 2. Results for the QCD equation of state. Core is in $M_{\odot}$, Radius in $\mathrm{km}$, time in $\mu \mathrm{s}$, density in $\mathrm{gm} / \mathrm{cm}^{3} .6 .40(14)$ means $6.40 \times 10^{14}$. The static neutron star mass for QCD is 2.34 solar masses.

\begin{tabular}{||c|c|c|c|c|c||}
\hline Core & bounce & Init. rad. & Fin. dens. & Fin. rad. & Time \\
\hline 0.75 & yes & 70.88 & $6.40(14)$ & 8.21 & 2175 \\
1.00 & yes & 78.01 & $7.80(14)$ & 8.48 & 2145 \\
1.38 & barely & 86.87 & $7.64(14)$ & 9.50 & 2110 \\
\hline
\end{tabular}

Table 3. The emitted and received (difference due to redshifts) directly radiated MEMP energy of the QCD and Argonne (ARG) EOS. Units are in ergs.

\begin{tabular}{||c|c|c||}
\hline Core & Emitted Energy & Received Energy \\
\hline QCD 0.75 & $1.07(43)$ & $1.07(43)$ \\
QCD 1.00 & $1.47(43)$ & $1.47(43)$ \\
QCD 1.3806 & $2.05(43)$ & $1.98(43)$ \\
ARG 0.75 & $1.27(43)$ & $1.27(43)$ \\
ARG 1.0825 & $1.82(43)$ & $1.76(43)$ \\
\hline
\end{tabular}

In the collapse studies, whenever a star goes below three times $a_{\mathrm{g}}$, we solve for the radius as a function of proper time by means of the Taylor expansion

$a(\tau)=a(t)+\frac{\mathrm{d} a}{\mathrm{~d} t}(\tau-t)+.5 \frac{\mathrm{d}^{2} a}{\mathrm{~d} t^{2}}(\tau-t)^{2}$.

So $a(\tau)$ is reconstructed from three times $a_{\mathrm{g}}$ to either the bounce radius or twice $a_{\mathrm{g}}$, for the purposes of calculating the MEMP spectrum.

It is also of interest to see the kinetics of black hole formation. Unexpectedly, we find that once the maximum core mass allowing hydrodynamic bounce is exceeded, the black hole is formed independently of the nuclear EOS. Thus, for example, the radius function a $(\tau)$, infall speed $v_{r}(a)$, and acceleration function $\operatorname{acc}(\tau)$ are all identical for a 1.5 solar mass core collapse, whatever nuclear EOS is used. Thus the MEMP of black hole formation is a universal signature. Though this has been derived in an effective gravitational collapse model, we believe this is a true theorem in physics. It is of interest then, whether the introduction of stellar rotation also reintroduces nuclear EOS dependence for black hole MEMP. It is probably true that once the core mass exceeds the neutron star dynamical mass, whether it is rotating or not, the black hole MEMP is a universal function. In Fig. 7, we give the kinetics of a 1.5 solar mass core collapse and a 5.0 solar mass core collapse, along with an example of a core bounce.

\section{Results}

The code logic is given in Fig. 5. Following Arnett (1977), the iron core begins collapse with a fairly uniform density of $10^{12} \mathrm{gm} / \mathrm{cm}^{3}$ and initial infall speed $v / c \sim \frac{2}{3} \times 10^{-2}$. After choosing the mass of the core, the initial radius is determined.
Table 4. Results for the Argonne EOS. The static neutron star mass for Argonne is 2.10 solar masses.

\begin{tabular}{||c|c|c|c|c|c||}
\hline Core & bounce & Init. rad. & Fin. dens. & Fin. rad. & Time \\
\hline 0.75 & yes & 70.88 & $1.60(15)$ & 6.05 & 2195 \\
1.08 & barely & 80.10 & $1.29(15)$ & 7.36 & 2144 \\
\hline
\end{tabular}

Table 5. 1.5 and 5 core mass collapse.

\begin{tabular}{||c|c|c|c||}
\hline Core & Initial rad. & Time & Rad energy \\
\hline 1.5 & 89.3 & 2142 & $2.66(43)$ \\
5 & 133.40 & 2001 & $7.60(43)$ \\
\hline
\end{tabular}

In Fig. 7, we present the results we obtained for the stellar surface for core masses of 1.5 and 5.0 solar masses, and in Fig. 8 the results for the energy spectrum of the emitted electromagnetic radiation, for the Argonne EOS (for a core of 1.0825 solar masses) and QCD (for a core of 1.3806 solar masses) EOS cases, and for core masses of 1.5 and 5.0 solar masses (independent of EOS).

\section{Conclusion}

We have presented an effective gravitational collapse model that is thought to include the essential physics: spherical radial infall in the Schwarzschild metric of a homogeneous core of an advanced star possessing a giant magnetic dipole moment. The electrodynamic equations are represented by the approximation of magnetohydrodynamic material response, which will be an excellent approximation due to the high electrical conductivity. Any nuclear EOS can be used, and we chose two which have high interest: the "Argonne" AV14+UVII which represents the typical phenomenological nuclear EOS and the QCD EOS. Surprisingly, the maximum core mass that bounced (the dynamic neutron star limit) is rather small using the phenomenological EOS.

The main result of the paper is the calculation of the MEMP from the collapse. Both the total energy and its spectral characteristics are derived. In cases where the core collapse is greater than the dynamical neutron star mass, the MEMP is the only electromagnetic experimental signature. It is hoped that this will spur activity to develop a suitable experimental receiver ${ }^{1}$. To answer the question as to whether the surrounding material from the collapsing object will quench or change an observational signal is clearly complicated, and it requires a detailed calculation in transport theory using information on the supernova models that vary tremendously on their precursor environment, which is beyond the scope of the present paper.

\footnotetext{
${ }^{1}$ Due to the peak of the spectral curve around the wavelength $\sim 2 \mathrm{~km}$, the receiver will have to be a satellite able to detect a broadband spectrum within the electromagnetic pulse. The additional benefit is that terrestrial signals in this frequency regime cannot penetrate earth's ionosphere, thereby reducing spurious man-made noise.
} 


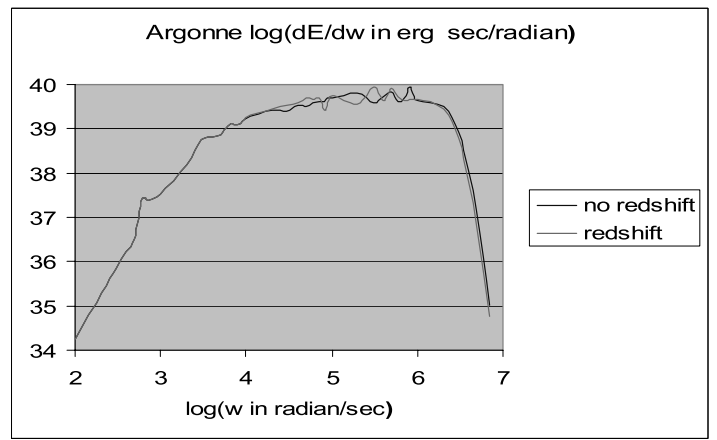

(1.5 solar mass) $\log (\mathrm{dE} / \mathrm{dw}$ in $\mathrm{erg} * \mathrm{sec} /$ radian $)$

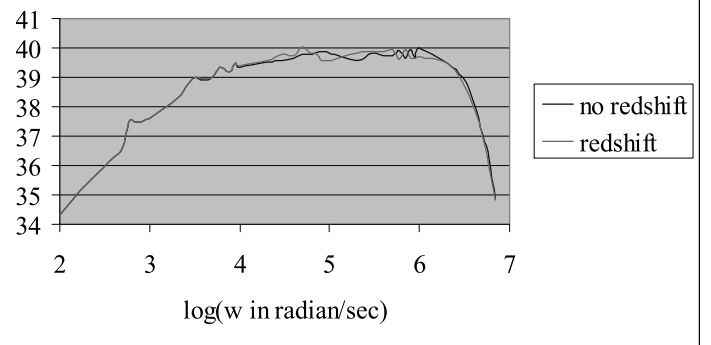

\section{QCD $\log (\mathrm{dE} / \mathrm{dw}$ in erg*sec/radian)}

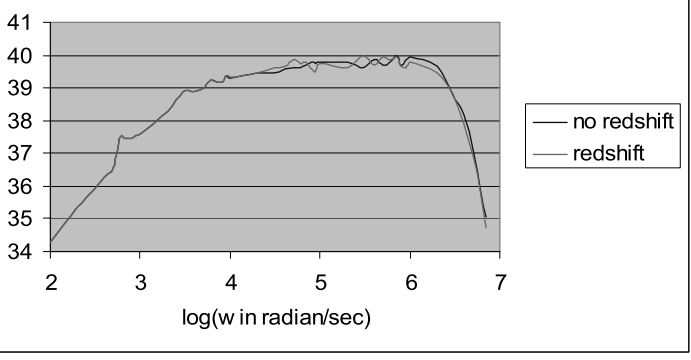

(5 solar mass) $\log (\mathrm{dE} / \mathrm{dw}$ in erg sec/radian)

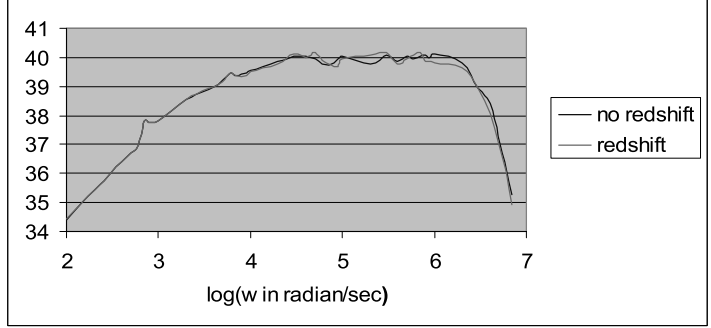

Fig. 8. Spectrum of the radiated energy, for the Argonne (for a core of 1.0825 solar masses) and QCD (for a core of 1.3806 solar masses) equations of state, and for a core mass of 1.5 and 5 solar masses, for the initial conditions mentioned in the text.

The existence of two classes of neutron star masses leads to the situation where a stellar core greater than the dynamical mass, but less than the static mass, collapses to form a black hole, thus producing black holes less massive than the mass limit of neutron star stability. For example, a black hole of 1.6 solar masses can exist. This possibility sheds light on one of the most perplexing problems of astrophysics: how do black holes of stellar masses form? As envisioned here, a core more massive than the dynamical mass collapses and forms a black hole. It may now accrete the remaining outlying surface matter to produce a whole continuum of stellar mass size black holes.

After this paper was submitted for publication, we were informed of the work of Hanami (1997). This author attempted to explain gamma-ray bursts using the change in the magnetic field of a collapsing stellar object. Unfortunately, there are several problems associated with this work. Hanami did not consider the fact that the star radiates continuously throughout the collapse trajectory and his numerical solution violates Maxwell's equations. He used a zero pressure EOS, which will not give the correct surface acceleration and core remnant. In order to compute the total energy, the gravitational redshift must be properly included. The calculation of the gravitational redshift is a very difficult problem as explained in the present paper, because, as the star collapses and radiates, each instantaneous stellar configuration has a different redshift. In order to do this, one needs to employ multiple Roll-on-roll-off mathematical functions in the complex energy plane, correct for the gravitational redshift and add the spectrums incoherently. Hanami did not consider this and simply calculated the redshifted power at the initial collapse configuration, to obtain his result. At the bottom of page 688 and top of page 689, Hanami (1997) alludes to the fact that his solution is the quasi-normal ringing (QNR), but his eigenfrequency is incorrect (QNR have precise discrete eigenfrequencies). Also his time dependence does not correspond to any known quasi-normal mode damping.

\section{References}

Arnett, W. D. 1977, Seventh Texas Symp. on Relativistic Astrophys.

Baym, G., Pethik, C., \& Sutherland, P. 1971, ApJ, 170, 299

Bocquet, M., Bonazzola, S., Gourgoulhon, E., \& Novak, J. 1995, A\&A, 301, 757

Cunningham, C. T., Moncrief, V., \& Price, R. H. 1978, ApJ, 224,643

Fryer, C. L., \& Heger, A. 2000, ApJ, 541, 1033

Hanami, H. 1997, ApJ, 491, 687

Hod, S. 1999, Phys. Rev. D, 60, 104053

Hod, S. 2000a, Phys. Rev. Lett., 84, 10

Hod, S. 2000b, Phys. Rev. D, 61, 064018

Iyer, S. 1987, Phys. Rev. D, 35, 3632

Joshi, P. S. 2000, gr-qc/0006101

Kislinger, M., \& Morley, P. D. 1978, ApJ, 219, 1017

Landau, L. D., \& Lifshitz, E. M. 1975, The Classical Theory of Fields (Pergamon Press)

Leaver, E. W. 1986, Phys. Rev. D, 34, 384

Morley, P. D., Dib, C., \& Schmidt, I. 2000, USM-TH-73

Price, R. H. 1972, Phys. Rev. D, 5, 2419

Straumann, N. 1992, Paul Scherrer Institute Proceedings 92-05, ISSN 1019-6447

Weinberg, S. 1972, Gravitation and Cosmology (John Wiley)

Wiringa, R. B., Fiks, V., \& Fabrocini, A. 1988, Phys. Rev. C, 38,1010 